La Revue

des Droits

de l'Homme

\section{La Revue des droits de l'homme}

Revue du Centre de recherches et d'études sur les droits fondamentaux

$11 \mid 2017$

Revue des droits de l'homme - $\mathrm{N}^{\circ} 11$

\title{
Entretien avec Olivier de Frouville, Professeur de droit international public à l'Université Panthéon- Assas, Membre du Comité des droits de l'Homme des Nations Unies
}

Sophie Grosbon

\section{(2) OpenEdition \\ Journals}

Édition électronique

URL : http://journals.openedition.org/revdh/2949

DOI : $10.4000 /$ revdh.2949

ISSN : 2264-119X

Éditeur

Centre de recherches et d'études sur les droits fondamentaux

Référence électronique

Sophie Grosbon, « Entretien avec Olivier de Frouville, Professeur de droit international public à

I'Université Panthéon-Assas, Membre du Comité des droits de l'Homme des Nations Unies », La Revue

des droits de l'homme [En ligne], 11 | 2017, mis en ligne le 16 janvier 2017, consulté le 08 juillet 2020.

URL : http://journals.openedition.org/revdh/2949 ; DOI : https://doi.org/10.4000/revdh.2949

Ce document a été généré automatiquement le 8 juillet 2020 .

Tous droits réservés 


\title{
Entretien avec Olivier de Frouville, Professeur de droit international public à l'Université Panthéon- Assas, Membre du Comité des droits de l'Homme des Nations Unies
}

\author{
Sophie Grosbon
}

$\left.11^{\circ}\right)$ L'année 2016 était l'année des 10 ans du Conseil des droits de l'Homme des Nations Unies, organe qui en 2006 a remplacé la Commission des droits de l'Homme. Quelles sont les principales différences entre ces deux institutions? Qu'est-ce qui vous parait constituer un progrès en matière de droits de l'Homme entre les deux institutions? Quelles sont les principales lacunes du Conseil des droits de l'Homme? Quels sont les principaux défis de cette institution aujourd'hui?

2 La création du Conseil des droits de l'homme a incontestablement constitué une évolution positive : le Conseil est un organe subsidiaire de l'Assemblée générale (et non plus un organe subsidiaire du Conseil économique et social), il siège plus souvent (trois sessions par an au lieu d'une, plus des sessions spéciales dont la convocation a été facilitée).

3 De plus, les principaux acquis de la Commission ont été pour l'essentiel conservés : droit de participation des ONG; point de l'ordre du jour consacré aux situations de violations des droits de l'homme; procédures spéciales qui portent à la fois sur des thèmes (procédures thématiques) et sur des pays particuliers (géographiques). À cela a été ajoutée une forme d'examen par les pairs, l'Examen périodique universel, que beaucoup considèrent comme le nouveau "joyau de la couronne", ce qui est discutable.

4 Cela dit, le Conseil a aussi hérité de quelques-unes des tares de la Commission. À l'époque, les États prétendaient condamner la "politisation" de la Commission, un terme auxquels ils ne prêtaient d'ailleurs pas toujours le même sens. Bien sûr, cette 
“politisation" se retrouve au sein du Conseil, puisqu'elle est inhérente à un organe intergouvernemental. Il ne faut pas espérer "dépolitiser" le Conseil. Par ailleurs, le Conseil a connu la même inflation que la Commission au cours des années - en pire. Son activité est devenue frénétique et, pour beaucoup, difficilement lisible.

5 Le défi principal est de dépasser le modèle actuel, qui privilégie justement le débat politique sur les droits de l'homme, ce qui contribue aux tensions et aux confusions. Juridiciser davantage la question des droits de l'homme est la seule manière de la dépolitiser : il faut que les États jouent leur rôle, qui est de faire pression sur leurs "pairs" pour mettre en œuvre les droits de l'homme, à travers les décisions prises par des experts ou des juges. C'est le sens profond de l'idée de garantie collective, que l'on retrouve dans les principaux traités en matière de droits de l'homme. Tant qu'on n'aura pas franchi ce pas, le système restera certes utile, mais aussi toujours en dessous des attentes qu'il suscite.

$\left.62^{\circ}\right)$ L'année 2016 était également l'année des 50 ans des Pactes de New York. Qu'est ce qui, selon vous, a principalement changé en matière de protection des droits de l'Homme au Comité des droits de l'Homme et au Comité des droits économiques, sociaux et culturels depuis 50 ans?

7 D'abord la qualité du contrôle exercé par les comités est sans commune mesure avec ce qu'il était à ses débuts. À partir du début des années 90, surtout, les Comités ont commencé à développer des méthodes de contrôle beaucoup plus contraignantes pour les États, avec notamment l'adoption d'observations finales à l'issue des dialogues. Auparavant, les comptes rendus analytiques de séance étaient la seule trace du dialogue, à présent, il y a ce document qui sert de référence, avec des conclusions et recommandations précises et qui peut faire l'objet d'un suivi d'un rapport à l'autre.

8 En 50 ans, il y a aussi eu le développement d'une jurisprudence impressionnante. Ce développement s'est effectué pour le Comité des droits de l'Homme essentiellement à travers ses "constatations" (à l'issue de l'examen des plaintes individuelles en vertu du Protocole facultatif au Pacte) puis ses "observations générales" (qui codifient et développent la jurisprudence du Comité sur un article du Pacte). Le Comité des droits économiques, sociaux et culturels, de son côté, s'est appuyé surtout sur l'examen des rapports et sur les observations générales : grâce à celles-ci, il a développé de manière impressionnante le contenu des droits du Pacte et en a préparé la pleine justiciabilité. Le Comité a fait de ce point de vue un travail essentiel et précurseur. Sans ce travail, il aurait été impossible de négocier l'adoption d'un nouveau protocole facultatif pour connaître de plaintes individuelles (adopté le 10 décembre 2008, entré en vigueur le 5 mai 2013, 22 États parties, dont la France).

9 Enfin, le troisième développement majeur que l'on peut relever est la croissance du "système des organes de traités". Avec l'adoption de nouvelles conventions, le nombre de comités a augmenté de manière considérable : de 3 comités, on est passé à 9 comités (correspondant à 9 conventions de base), plus un sous-comité contre la torture. Ce développement est donc spectaculaire et l'on doit d'abord s'en féliciter, même si de nouveaux problèmes en découlent, car désormais le système est devenu plus difficilement lisible et utilisable pour les États et les victimes. Les réflexions actuelles portent justement sur la manière de mieux organiser ce système afin d'une part de le rendre plus accessible et d'autre part d'alléger la charge qu'il fait peser sur les États en termes de "reporting", sans pour autant diminuer l'impact de leurs recommandations. 
$\left.103^{\circ}\right)$ Pensez-vous qu'aujourd'hui les droits économiques, sociaux et culturels sont reconnus comme des droits de l'Homme aussi importants que les droits civils et politiques ou continuent-ils à être marginalisés au sein des organes des traités ?

11 Ils sont loin d'être marginalisés, mais je dirais qu'ils ne sont toujours pas à égalité exacte avec les droits civils et politiques. Le Comité des droits économiques sociaux et culturels (CESCR) a donné, au fil de ses observations générales, un contenu à ces droits et aux obligations qui en découlent pour les États. En ayant à l'esprit ces travaux, il ne devrait donc plus être possible d'affirmer aujourd'hui sérieusement que ces droits sont "programmatoires", ou de maintenir par exemple les vieilles distinctions entre "droitslibertés" (qui ne coûteraient rien) et "droits-créances" (qui seraient coûteux) ou même entre différentes "générations" des droits de l'Homme (avec pour conséquence implicite que les deux dernières générations seraient moins "juridiques" que les deux autres). Au-delà du CESCR, les instruments catégoriels traitent aussi des droits économiques, sociaux et culturels, ce qui contribue à en "banaliser" l'interprétation. Je crois que s'il existe encore des réticences, elles tiennent davantage à la préoccupation qu'ont certains experts de maintenir à chaque comité un périmètre bien défini. Par exemple, dans le cas du Comité des droits de l'Homme, certains craignent qu'une interprétation trop large du droit à la vie qui incorporerait la dimension économique, sociale et culturelle de ce droit, détournerait le Comité de ce qui devrait être ses priorités, à savoir, entre autres, les exécutions arbitraires ou la peine de mort. Je pense de mon côté qu'il est nécessaire que les comités aient une interprétation cohérente fondée sur l'indivisibilité, ce qui implique par exemple pour le Comité des droits de l'Homme de reconnaître la dimension économique, sociale et culturelle du droit à la vie ; mais qu'en même temps les comités doivent davantage chercher à rendre leurs actions complémentaires et éviter autant que possible les duplications. Par exemple, il est nécessaire de généraliser les références croisées dans les observations finales, voire même au stade des listes de questions, à tout le moins lorsque les examens sont raisonnablement rapprochés dans le temps. Par exemple, si le CESCR a traité d'une situation de privation de l'accès à l'eau comme ressortant des articles 11 et 12 du PIDESC, il ne me semble pas nécessaire que le Comité des droits de l'Homme revienne sur la même situation trois mois après au titre de l'article 6 du PIDCP - à moins bien sûr que des développements importants aient eu lieu entretemps, l'important étant de rester flexibles et réactifs et en tout cas de viser l'effectivité.

\section{$4^{\circ}$ ) Quels sont les chantiers du futur?}

Il faut d'abord préserver les acquis. Dans la période qui s'ouvre, cela n'a plus rien d'évident. Les attaques contre le système de protection des droits de l'Homme ont déjà été nombreuses par le passé, à chaque fois le système a continué de grandir et de se renforcer. La réaction des États-Unis au 11 septembre a ouvert une période de régression en matière de droits de l'Homme sur le plan mondial. Les printemps arabes ont marqué une courte éclaircie, mais la dégradation de la situation en Syrie, la montée des tensions entre l'Occident et la Russie, l'afflux des réfugiés en Europe et la crise de l'Ukraine ont favorisé la montée en puissance des populistes et des démagogues qui font du rejet de l'autre leur fonds de commerce. Il est certain aujourd'hui que les droits de l'Homme - en tout cas entendu dans une perspective authentiquement universelle et juridique et non comme un discours purement politique teinté d'impérialisme - ne sont plus la priorité. Il faudra donc tenir bon dans les années qui viennent pour préserver les principaux acquis du système, à savoir ces îlots d'indépendance que sont, aux 
Nations Unies, les procédures spéciales et les organes de traité. Aujourd'hui, les ONG et les défenseurs des droits de l'Homme ont tendance à considérer le système de protection des droits de l'Homme des Nations Unies comme s'il allait de soi, alors qu'il est le fruit de 70 années de combats pour les droits de l'Homme. Les ONG et les victimes ne peuvent pas se contenter d'être les "usagers" du système; elles doivent se l'approprier, le défendre et faire des propositions pour l'améliorer. D'une certaine manière, le système est aujourd'hui victime de son succès : le Conseil des droits de l'Homme s'est emballé et les sessions sont de plus en plus chargées; le nombre de procédures spéciales ne cesse de croître ; le système conventionnel lui-même comprend dix organes, comme je le disais plus haut et désormais chaque comité dispose d'une procédure de plaintes... Tout cela devient illisible à la fois pour les États et les victimes. C'est comme une courbe en cloche : l'accroissement des procédures a jusqu'à un certain point fait augmenter l'effectivité et l'impact du système. Si l'on continue, on risque d'aboutir à un système ingérable qui deviendra petit à petit insignifiant. Les organisations de la société civile et les États favorables aux droits de l'Homme (il en reste!) ont donc un rôle important: il faut qu'ils proposent à terme un projet ambitieux dont on puisse expliquer le sens et les avantages aux opinions publiques. L'idée d'une Cour mondiale des droits de l'Homme doit être explorée plus avant. De même, l'idée d'une Commission ou d'un Conseil qui renouerait avec la proposition initiale de René Cassin - à savoir une commission d'experts indépendants - devrait également être étudiée sérieusement. L'évolution des systèmes de protection des droits de l'Homme montre que ceux-ci gagnent en effectivité lorsque l'expertise juridique est mise au centre du dispositif d'interprétation et de décision, tandis que les organes politiques se concentrent sur la mise en œuvre. Il faut suivre cette voie et la développer, avec de la créativité, mais aussi de la flexibilité et un sens des réalités politiques.

\section{AUTEUR}

\section{SOPHIE GROSBON}

Sophie Grosbon est Maîtresse de Conférences en droit public à l'Université Paris Nanterre 